\title{
Placement avec un rendement intéressant et un taux garanti
}

\section{Helvetia Swiss Trend, I'assurance-vie liée à un indice}

\section{Qu'est-ce que Helvetia Swiss Trend?}

Helvetia Swiss Trend est une assurance-vie liée aux tendances de l'indice des actions SMI. Le SMI augmente, votre placement augmente. Le SMI chute, votre capital, indépendant des cours de la Bourse, est tout de même garanti et bénéficie du taux annuel garanti.

Helvetia désire renouer avec les succès des années nonante. A cette époque trois tranches de ce produit avaient été lancées, produit qui avait permis de réaliser, malgré le crash boursier du début du siècle, un rendement d'au moins $6 \%$ annuellement.

\section{Vos avantages en bref}

- Perspectives de rendement intéressantes grâce à la participation de la tendance de l'index du marché suisse SMI

- Protection contre les baisses de la Bourse

- Taux minimum garanti du capital d'épargne

- Versement du capital exonéré fiscalement pour autant que les conditions de l'administration fiscale fédérale soient remplies (versement après 60 ans, conclusion avant 66 ans, durée minimale 5 ans)

\section{Historique du succès}

Déjà plusieurs produits Helvetia Swiss Trend ont été placés sur le marché avec succès:

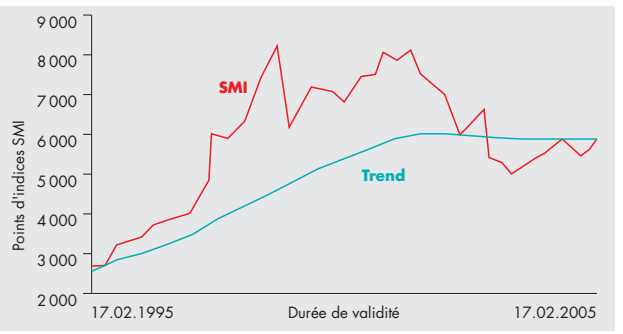

Exemple: Patria Swiss Trend II a atteint un rendement moyen de 8,5\% par année
Attention Souscription du 9.7. au 7.9.2007 Ce produit est uniquement disponible par tranche. La souscription a débuté le 9 juillet et se termine le 7 septembre 2007. Une nouvelle tranche n'est pour l'instant pas prévue.

Profitez vous aussi de cette occasion unique et commandez encore aujourd'hui votre offre personnelle au moyen du talon réponse joint.

\section{helvetia $\Delta$}

\section{Talon réponse}

Prénom / Nom

Adresse

NPA / Lieu

Date de naissance

Télephone privé / cabinet

Atteignable le plus facilement (heure)

Adresse e-mail

Prière de m'envoyer une offre pour l'Helvetia Swiss Trend. Montant: Fr.

○ Je désire un conseil personnalisé.

Prière de me téléphoner.

- Je suis intéressé aux thèmes suivants:

\footnotetext{
O Placement de capitaux

O Caisse de pension LPP

O Pilier $3 a$

O Assurance responsabilité prof.

O Retraite anticipée

O Protection juridique
}

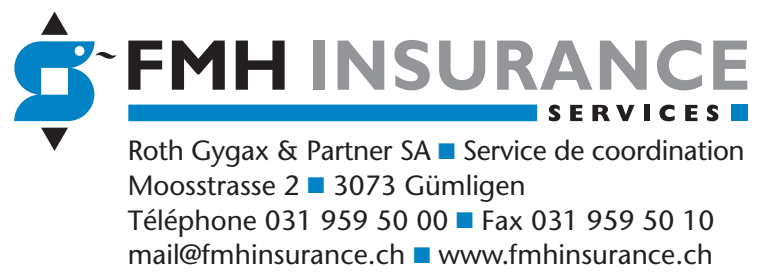

